\title{
Design of an Automatic Double Threaded Trampoline Webbing Machine
}

\author{
Shi Feng and John P. T. Mo \\ School of Engineering, RMIT University, Melbourne, Australia \\ Email: shi.feng@rmit.edu.au,john.mo@rmit.edu.au
}

\begin{abstract}
The main concept of this paper is to design and develop an automatic webbing machine for Olympic grade trampoline manufacture. The Australian company adopts a manufacturing process that produces double threaded trampoline net which has much better quality than the competitors. During the original weaving process, the wrap and the weft are continuous, so that the adopt double thread weaving is maintained. This specific weaving function leads to the popular weaving machine which are applied widely in textile industry cannot be introduced. Two manual machines were designed to manufacture trampoline nets but they limited the capacity of production. This paper focuses on the evaluation of the performance in manufacturing of the trampoline nets with pneumatic device controller which is based on the evidence and related material investigations. After designed in a CAD model, a proportionally scaled down prototype was manufactured using 3D printing technology. Preliminary test and evaluation were successful offering practical data and new improvement ideas for actual application and commercialization.
\end{abstract}

Index Terms - Double threaded webbing, trampoline net, pneumatic automation

\section{INTRODUCTION}

Company $\mathrm{T}$ is Australia's leading trampoline manufacturer making Olympic grade trampolines for commercial and leisure use. The manufacturing process was invented half a century ago and is a unique process that guarantees quality and durability of the products, compared to many other trampoline manufacturers in the world.

Rapid development of the business and high demand of the quality product require increase in manufacturing capacity. However, the current manufacturing process, although assisted by a custom-designed weaving machine, is largely a manual process. It requires a lot of skills and adjustments to ensure each threading action is complete correctly. The manufacturing output is limited by the capacity of the machine and availability of skilled operators.

The research is to investigate the design and manufacture of an automatic machine that can produce the same (or better) quality trampoline mat without human intervention.

Manuscript received May 7, 2018; revised July 28, 2019.

\section{LITERATURE REVIEW}

Due to the special manufacturing method used in the factory, literature related to continuous double-threads webbing technology is rare. The literature review has been broadened to weaving technologies which are similar to webbing technologies. By comparing with these two technologies, some insight of the web manufacturing process and automation requirements can be identified.

\section{A. Principles of Weaving}

There are three generally accepted weaves, plain weave, twill weave and satin weave. Texture of the fabrics will vary from different weaves.

Plain weave is the simplest weave in the above weaves [1]. It works by repeating one-over, one-under interfacing for both weft and warp. The texture of plain weaved fabrics is the same on both top and bottle sides. Plain weaves only require two harnesses, but it will vary according to warp density. Due to the one-over, oneunder interfacing, the level of yarn crimp in the plain weave is the highest compared to other weaves.

Twill weave is produced by a stepwise progression of the warp yarn interfacing [2]. The interfacing pattern of the warp yarn starts at different weft. A diagonal pattern is produced by a progressive increment in the fabrics, called twill line. According to the direction of the twill line, there are two different types of twill weaves: right hand twill and left hand twill. In the right hand twill, twill line moves from lower left to upper right. In the left hand twill, twill line moves in reverse. The result is that the twill weaved fabric has right hand on the surface and have left hand on the back. Twill angle can vary too. If the twill line angle is 45 degrees, the design is called common twill. If it is greater than 45 degrees, the design is called steep twill. Twill line less than 45 degree is called reclining twill.

In the satin weave, one yarn stays over or under more than one yarns, this yarn is considered to have a float [3]. If a yarn is on the top surface of the fabric, it is called a "raiser", otherwise, it is called a "sinker". Satin weave requires at least 5 harnesses and the satin weave is named after the minimum number of harnesses, for example, 5harness satin, 7-harness satin. Satin weaves can be classified as warp-faced or filling faced, if the long warp float is on the top surface of the fabric then the design is 
called warp-faced. If the long filling float is on the top surface, the design is called filling faced [4].

Twill and satin weave methods are more suitable for forming patterns on the fabrics and hence are not useful for plain web requirements of trampoline manufacture. The principle of weaving in this case is based on plain weave.

\section{B. Filling Insertion System}

The weaving machines can be classified as shuttle and shuttles weaving machines. The shuttle looms have been used for centuries to make woven fabrics. However, in the last century, other weaving machines using different filling insertion mechanisms such as air, projectile, rapier and water replaced shuttle weaving machines. These weaving machines called shuttle-less looms or shuttleless weaving machines [5].

Many filling insertion methods have been patented. For example, Shaw and Salomez [6] invented a method to monitor filling insertion in a weaving machine. The invention monitored the filling insertion process and the time it took. It is therefore noted that effectiveness of fill insertion method determines efficiency of the machine.

\section{Pneumatic Systems}

Increasingly, pneumatics is used in modern automated systems. Compared to motors and actuators, the benefits of pneumatic systems are lower cost, higher variability and safer. Most of industrial pneumatic systems can offer pressure from about 5.5 to $6.9 \mathrm{MPa}$. The pneumatic system can be designed with the standard cylinder and components and can be operated with a simple switch control [7]. It has the advantages that:

a) It has a long service life and requires less maintenance because gas is compressible and the equipment will not be damaged by the impact.

b) After the power interruption, pneumatic system can still operate a short time because the pressure of reservoir of the compressor will last for some time.

c) Compared to the hydraulic system, pneumatic systems are smaller and can be overloaded.

Most pneumatic system can be controlled directly by valves which have different functions. Pneumatic valves can be divided into three main categories defined by the function, which are directional control, flow control and pressure control [8]. They can be further divided into those valves which control the airflow to carry out a power function and those which perform a control function. The latter includes several specialised valve types which only perform a logic function in control circuits [9]. In recent years, many new applications have been replaced by electronic control system pneumatic logic, because digital system, its small, low cost, high precision and powerful functionality.

Hence, it is practical to replace some mechanisms by more straight forward pneumatic actuators and control systems.

\section{3D Printing Technology}

$3 \mathrm{D}$ printing, also known as additive manufacturing, is used to produce 3-demensional objects. Main steps of using the $3 \mathrm{D}$ printing technology are, data generation, transferring data into the form that 3D printing machine can recognise, then use the computer controlled the 3D printing machine to generate successive layers of materials to create the desired objects [10].

With the development of the 3D printing technology, numbers of $3 \mathrm{D}$ printing processes are available to produce 3-demensional objects. Unfortunately, many 3D printing projects failed because of lack of appreciation of the cost-benefits relationship. A case study showed that the perceived value of timeliness of spare parts in a service oriented environment played an important role ensuring mission success [11].

Based on the materials being used in the processes and the way layers are deposited to make the parts, there are 6 different types of $3 \mathrm{D}$ printing processes. The most common 3D printing technology is the fused deposition modelling (FDM) [12]. The working principle of the FDM printer is to heat thermoplastic filament to its melting point and then extrude the melting materials layer by layer to create the designed three dimensional object.

The 3D printing facilities available to the research team has provided much flexibility in the design of components on the new machine.

\section{E. Micro-controller}

Arduino is an open source electronic platform. It uses a variety of microprocessors and controllers. The boards are equipped with sets of digital and analog input/output (I/O) pins that can be interfaced to various expansion boards and other circuits. The board feature serial communications interfaces, including USB, which can be used for loading program from computers. The microcontrollers are programmed by a language similar to $\mathrm{C}++$ [13].

Fatehnia et al [14] designed a system of automated double ring infiltrometer using an Arduino microcontroller and electronic accessories. The system was used for infiltration measurements and was not affected by sunlight. The system automatically detected when the steady state infiltration rate was reached and concludes the testing and stops measurements.

Shubert et al [15] used an open-source Arduino microcontroller to measure response latencies on keyboards They demonstrated the reliability, robustness, and precision of communication with Arduino as a serial response box was better than a keyboard. The setup was also flexible to be integrated with different sensors and actuators. With the Arduino micro-controller, the complex pneumatic sequences can be realized readily.

\section{ENGINEERING BASELINE}

The basic engineering information described below forms the underlying baseline for benchmarking the innovation in this project. The machine can be regarded as a plain weave machine with a rapier loom but it has a major difference, i.e. they have a double threads. The weft and warp in the machine are continuous.

Unlike the traditional weaving machines, the warp preparation is a much more complicated process. During 
the warp preparation, there are two sets of hooks at both sides of the machine. The hooks are mounted on a strainer which is tied to a roller. Figure 1 shows the hooks mounted on the strainer.

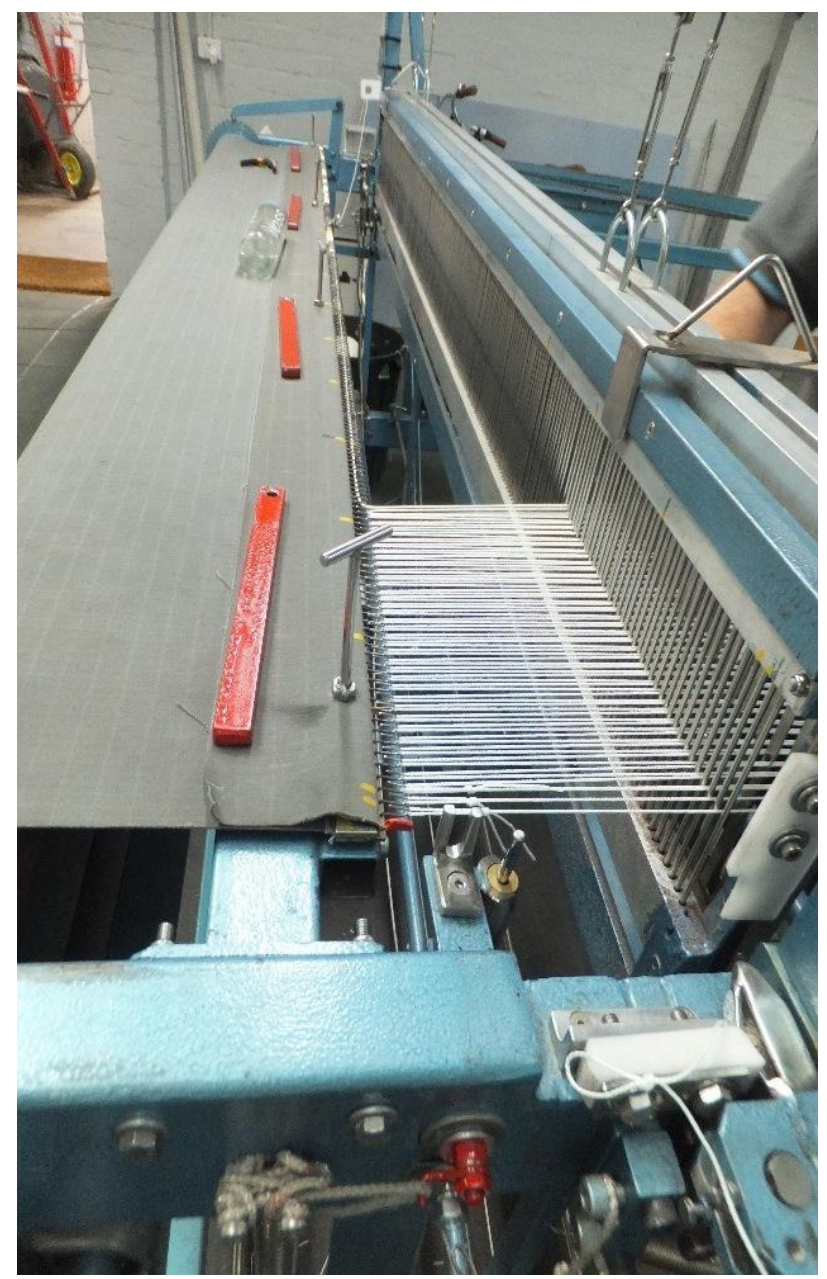

Figure 1. Hooks are mounted on the strainer at one end of the machine.

During the weaving process, a chain mechanism drives the roller on the weft insertion side roll to wind the manufacturing trampoline mats, also the roller on the other side will rotate simultaneously to feed the trampoline mats. In order to roll the trampoline mats, the worker have to tie the warp manually on both side of the hooks and this process is the warp preparation in the factory (Figure 2).

The warp preparation process has to be operated by skilled workers because the tension required to tie the warp on the hooks is hard to control. The worker needs to tie the weft thread on one of the hooks in one side, guide the warp thread through the eyes of the heddles, and move to the other side of the machine, using appropriate force to tie the thread on the opposite hook. This operation is repeated until all warp threads are tied on hooks. Therefore, the warp preparation in the factory is time consuming and prone to errors.

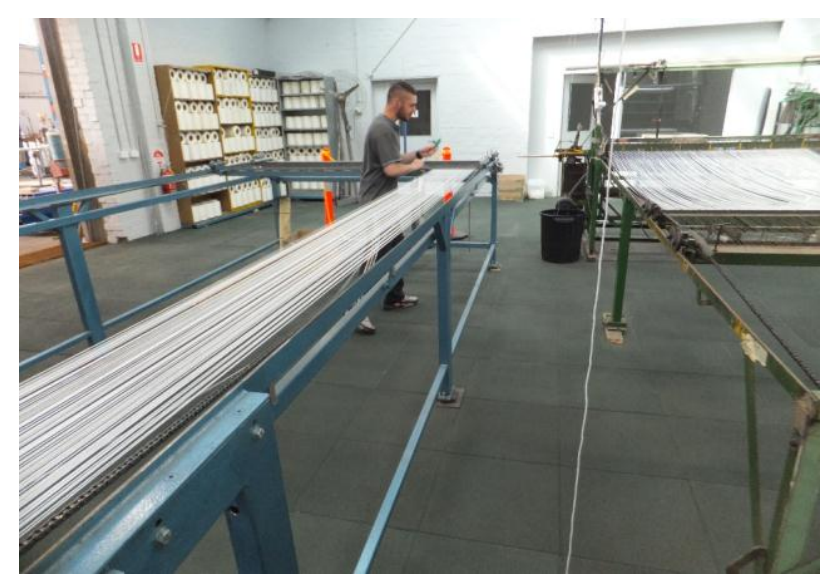

Figure 2. Warp preparation in the factory.

Next step is the weft insertion process. When the weft is picked from the weft package, rapier will carry the weft to cross the width of the warp. When the weft from the right side of the weaving machine hits the picking point of the weft, a shaft on the left side immediately move up to hook the weft, then the rigid rapier move back to the right side position with a spring mechanism. This movement doubles the weft thread. Then the reed in the machine will pull back by a spring mechanism to the initiating end. The back and front heddle frames change their positions. After shedding process, the worker winds the roller of the strainer to roll up the trampoline mat forward. At this point, first round of the weaving process has completed.

In this weaving process, rigid rapier plays an important role in guiding the weft thread, the working principle of the weft insertion process in this machine is to use a belt driving a set of wheels to contact and push the rapier forward when the worker rotates the control mechanism. If the rapier is not contacted with the wheels, it will be returned by a spring (Figure 3 ).

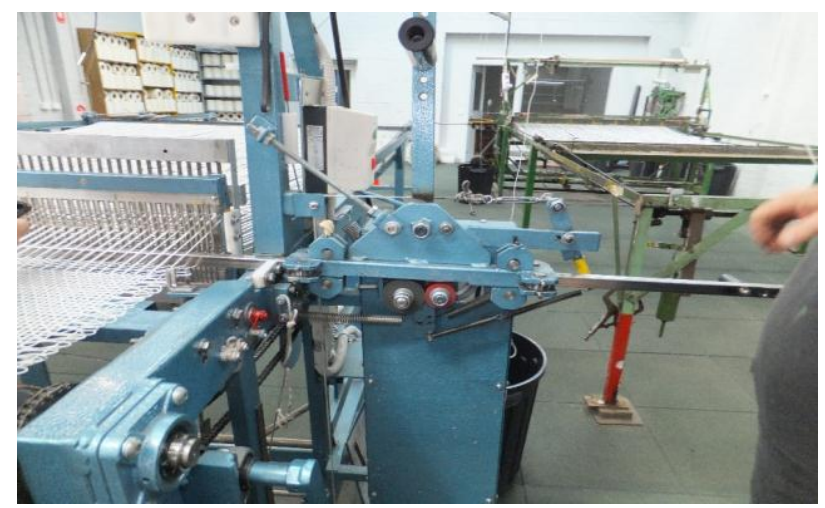

Figure 3. Rapier insertion mechanism.

For the shedding process, the machine is equipped with a tappet shedding mechanism. The worker rotates the bar to activate the tappet shedding mechanism in the bottom of the machine (Figure 4). 


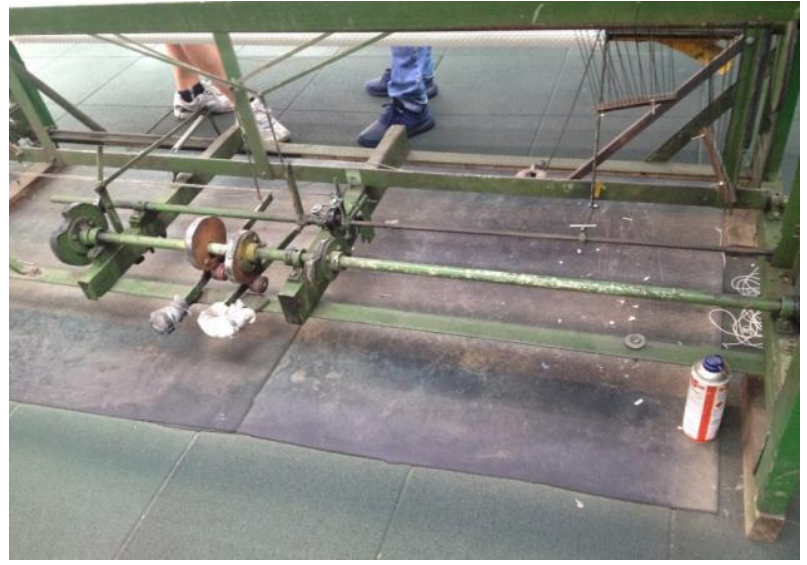

Figure 4. Tappet shedding mechanism at the bottom of machine.

Although tappet shedding is the simplest shedding mechanism, but it has a better production compared with other two main shedding mechanisms (Jacquard shedding, Dobby shedding). Meanwhile, tappet shedding is very suitable for manufacturing simple design products, and the installation cost is lower than others. The design pattern of the trampoline mats is simple, which is practical for the use of the plain weave and tappet shedding mechanism.

\section{Automated Machine Design}

Since the existing manufacturing machines are still being used in production, it is not possible to set aside one of the machines for a few months to be researched, re-designed and installed the new control system. It is proposed that Mr. Trampoline will build a new machine frame with the basic weaving features so that new components and control mechanisms can be built on it.

\section{A. Design Concept}

A preliminary study has shown that while automating the whole machine would be an ideal outcome, it would be better to limit to a few key functions at the start to gain more practical knowledge of the system and to identify potential issues in the integration of modern automation technologies to this type of mechanical actuated machines. If the first part is successful, an extension of the project can be developed to investigate automating the remaining parts of the machine.

This research focuses on how to change the weft insertion process to an automatic process. The warp preparation process will remain unchanged (i.e. still setup manually). Since the weaving mechanism of existing machines works well with manual intervention, there is no need for substantial re-design of the rapier, left and right shafts. The project will re-design the driving mechanisms with new electrical and pneumatic devices that can be controlled by a microcontroller.

\section{B. Project Plan}

The following table of work breakdown structure is developed on the basis of the input and outcome described previously.
TABLE I. WORK BREAKDOWN STRUCTURE OF DOUBLE THREAD MACHINE PROJECT

\begin{tabular}{|l|l|}
\hline Code & Description of work \\
\hline 100 & Semi-automated double thread weaving machine \\
\hline 100.1 & $\begin{array}{l}\text { Design and build new machine frame and non- } \\
\text { automated weaving features }\end{array}$ \\
\hline 100.1 .1 & Develop process and operations maps of new machine \\
\hline 100.1 .2 & $\begin{array}{l}\text { Identify and document existing features and } \\
\text { components }\end{array}$ \\
\hline 100.1 .3 & Manufacture new machine frame and basic features \\
\hline 100.2 & $\begin{array}{l}\text { Design and build new automated features and } \\
\text { components }\end{array}$ \\
\hline 100.2 .1 & 3D component designs \\
\hline 100.2 .2 & 3D printing of plastic components \\
\hline 100.2 .3 & $\begin{array}{l}\text { Manufacture (typically machining) of metal } \\
\text { components }\end{array}$ \\
\hline 100.2 .4 & Source commercial components \\
\hline 100.3 & Design and program microcontroller \\
\hline 100.3 .1 & Develop process sequence on new machine \\
\hline 100.3 .2 & Design and assembly of wiring \\
\hline 100.3 .3 & Design of human-machine control interface \\
\hline 100.3 .4 & Programming and debugging \\
\hline
\end{tabular}

The cost breakdown structure serves as a guide to all parties in this project to share work and intellectual property readily.

\section{Design Objectives}

Experience from the factory on the properties of the yarn is important to minimize potential problems. Two problems were focused in the first prototype design:

1. The rapier controlled manually may lead to missing hook, influences the efficiency. The rapier action will be changed to automatic. A motor drives the pulley, when the pulley touched the rapier, to drive the rapier move. However, the default rotational speed and the settled touching time will ensure the rapier moves same distance and arrive at the same position in each loop, to avoid not arrive the default location or exceed the default location.

2. The chain transmission system controls several operations manually, which leads to the weft intervals are not uniform, and the trampoline fabric is too tight when collected by the collect roller. The chain transmission system will be separated to reed control, heddle cross change control and roller rotation control. These controls will be automatic.

\section{Micro-controller Design}

Once the mechanical process is determined, coding of the micro-controller can be developed (Figure 5).

The Arduino program is basically a control loop scanning all necessary inputs and responding with appropriate outputs. The control loop can be described in 7 steps:

a) The rapier inserts from the right side to the left side.

b) The left shaft pushes up when the rapier head will arrive the left default location. 


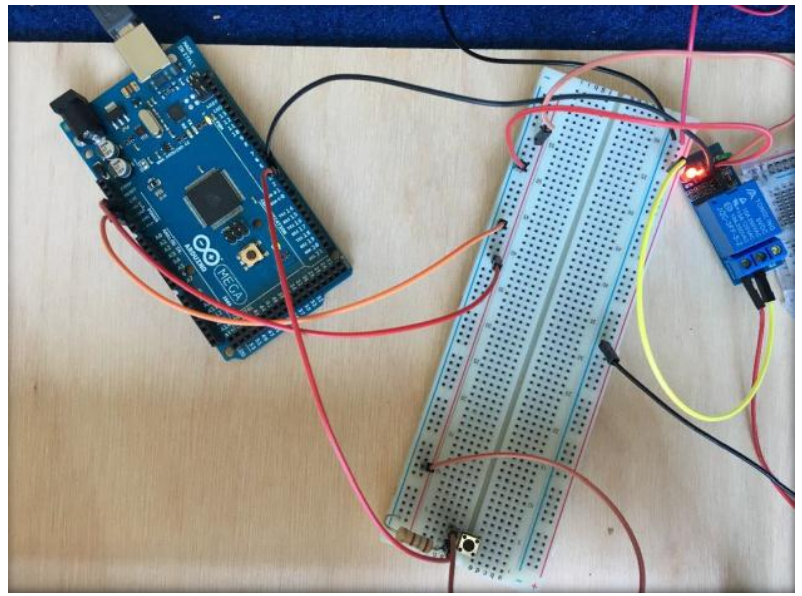

Figure 5. Arduino and prototype circuit.

c) The rapier is pulled back by the spring, when the pulley does not touch the rapier, the driving force to offset the spring force is disappeared. Then the rapier returns to the right initial position.

d) The reed pushes down, meanwhile, the left shaft and the right shaft push down together.

e) The reed is pulled back by the spring, meanwhile, the front and the back heddles cross change the position, and the right shaft pushes up to hold the weft.

f) The collect roller rotates 5 degrees to collect the trampoline fabric.

g) The whole loop is completed, it will return to the step (a) and repeat the circulation.

\section{DISCUSSION}

In this project, multiple engineering knowledge has been applied in our design. The CAD software Solidwork is used for drawing the prototype, Arduino is being applied as the primary controller. 3D printing technology is being used for printing the components in the model.

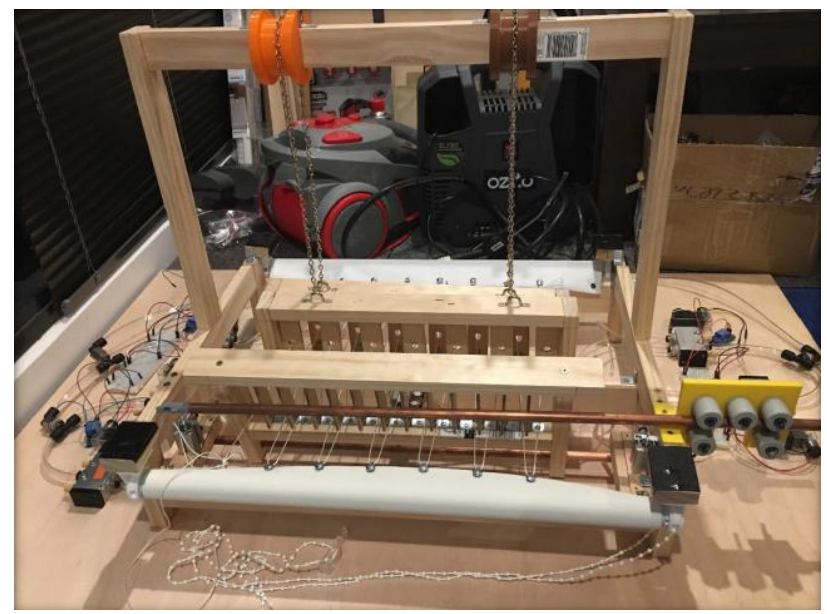

Figure 6. Model for testing effectiveness of the design.

To prove the design concept, a weaving machine model is made. In this preliminary design, the weft insertion process has been changed to a pneumatic control process. Shedding mats and rolling mechanisms are replaced to pneumatic devices. All the design components are assembled, all the Arduino control programs are proved to work well in the new design, the weaving process has been tested and it works successfully. As it is a conceptual model, it is used for illustration of key features that will be optimized in the final design. Figure 6 shows the conceptual model.

The original manual machine needs at least two technicians to operate. In the automatic machine, only one technician can control the whole machine. The technician can check all other details and control the machine any time with the machine switch.

Although the manufacturing speed of the prototype machine cannot be compared directly with the original manual machine, the decreasing of the waste time is obvious. The standard operation of the rapier inserting avoids repeating operation due to the manual error in missing hook.

Four situations are not included in this preliminary research:

a) Right meshes are not uniform because of the friction between the right shaft and the weft, which need to be held by fingers. This situation is planned to be fixed by two automatic fixes that installed on the left shaft and the right shaft. When the right sensor detects the right shaft hooked the weft, the right fix clamps the weft to keep the weft does not move. Similarly, the left fix will clamp the weft when the left sensor detects the left shaft hooked the weft, to avoid the weft moves.

b) Left meshes need to be closed the edge manually. A sword hook with a certain length will be installed at the left side of the machine. Each time after the left shaft is pushed down, the sword hook moves forward to hook the left mesh and collect them back. When this sword hook collected a certain of left meshes, another sword hook with the closing edge yarn passes through these meshes to complete closing edge.

c) The warp may cross overlap sometimes because of the friction and the static electricity. The initial idea is to add a yarn filter in front of the reed, keep the warp are flat after heddles cross changed position.

d) All warps are hooked manually before manufacturing. The manager of Mr. Trampoline said this step no need to add an automatic process, or maybe it already has a solution. Therefore, this problem will not be discussed in this research.

\section{CONCLUSION}

In this research, the working principles of the weaving processes like warp preparation, weft insertion, and shedding have been investigation as the basis for new design. Based on the size and shape of the original weaving machines in the factory, a weaving machine prototype is created. The design prototype is the foundation of the next manufacturing and assembling processes, since our assembly work has successfully completed in the end, the design prototype is proven to be a practical design. All the complex parts like rapier head, rapier support holder have been printed in the school 
laboratory. Although the components have some tolerances due to the precision limits of the 3D printing machines, but most of the print parts can be mounted on the design model, and the design model can operate all the weaving processes as expected, which means the design dimension of the components are reliable.

\section{REFERENCES}

[1] S. Adanur, Handbook of Weaving, CRC Press, 2000.

[2] K. Gandhi, W. Sondhelm, "Technical fabric structures - 1. woven fabrics," in Handbook of Technical Textiles: Second Edition. Elsevier Inc., 2015, pp. 63-106

[3] D. J. Jeremy, "The textile machinery collection," Technology and Culture, vol. 18, no. 1, pp.70-79, 1977.

[4] G. L. Rogers, "Textiles and textile machinery (Book Review)," Technology and Culture, vol. 1, no. 4, pp. 394-401, 1960.

[5] J. L. Conrad, S. Slater, "Drive that branch: the power loom," Technology and Culture, vol. 36, no. 1, pp.1-28, 1995.

[6] H. Shaw, H. Salomez, "Method and apparatus to monitor filling insertion in a weaving machine, with detection of an exact time at which the filling is actually severed," European Patent: US6273150, 2001.

[7] A. Parr, Hydraulics and Pneumatics: a Technician's and Engineer's Guide, 3rd ed., Elsevier Science, 2011.

[8] H. B. Wang, J. P. T. Mo, N .N. S. Chen, "On the estimation of onoff valve parameters for fuzzy logic control of programmable pneumatic actuators," Transaction of Institute of Measurement and Control, vol. 20, no. 5, pp. 211-220, 1998.

[9] R. M. Haney, Solenoid Control, Testing, and Servicing: a Handy Reference for Engineers and Technicians, New York: McGrawHill, 2013.

[10] J. R. Tumbleston, D. Shirvanyants, N. Ermoshkin, R. Janusziewicz, A. R. Johnson, D. Kelly, K. Chen, R. Pinschmidt, J. P. Rolland, A. Ermoshkin, E. T. Samulski, and J. M. Desimone, "Additive manufacturing. Continuous liquid interface production of 3D objects," Science (New York, N.Y.), 2015, vol. 347, no. 6228 pp. 1349-52.

[11] K. Ghaoui, J. P. T. Mo, "Application of three-dimensional printing technology to support services of defence systems," in Proc. The Spring Servitization Conference, 18-19 May, 2015, Birmingham, UK.

[12] A. Gebhardt, J. S. Hèotter, Additive Manufacturing: 3D Printing for Prototyping and Manufacturing, Hanser Publications, 2016.

[13] S. F. Barrett, Arduino microcontroller processing for everyone! Pub. Morgan and Claypool, 2013.

[14] M. Fatehnia, S. Paran, S. Kish, and K. Tawfiq, "Automating double ring infiltrometer with an Arduino microcontroller," Geoderma, 15 January 2016, vol. 262, pp. 133-139

[15] T. Schubert, A. D'Ausilio, R. Canto, "Using Arduino microcontroller boards to measure response latencies," Behavior Research Methods, 2013, vol.45, no. 4, pp.1332-1346.

Shi Feng graduated from RMIT with a Master degree in Manufacturing Engineering. He is currently a Research Fellow in RMIT specializing in the development of automated systems with flexible manufacturing processes such as 3D printing. During his research, he has initiated the investigation of the necessary processes in systems design and the effect of various automation technologies for different industry applications.

John P.T. Mo is professor of Manufacturing Engineering and formerly Discipline Head of Manufacturing and Materials Engineering at RMIT University, Australia. Prior to joining RMIT, he was Senior Principal Research Scientist in CSIRO and led research teams including Manufacturing and Infrastructure Systems. In his 11 years in CSIRO, his team worked on many large scale government and industry sponsored projects including electricity market simulation, infrastructure protection, wireless communication, fault detection and operations scheduling. He was the project leader promoting productivity improvement in furnishing industry and consumer goods supply chain. John has over 350 publications in referred journals, conferences and book chapters, 3 edited books, one monograph and 80 confidential reports. 